\title{
Spectrum Trading with Insurance in Cognitive Radio Networks
}

\author{
Haiming Jin, Gaofei Sun, Xinbing Wang \\ Dept. of Electronic Engineering \\ Shanghai Jiao Tong University, China \\ Email: \{jinhaiming, sgf_hb, xwang8\}@sjtu.edu.cn
}

\author{
Qian Zhang \\ Dept. of Comp. Sci. and Engin \\ HK Univ. of Sci. and Tech., HongKong \\ Email: qianzh@cs.ust.hk
}

\begin{abstract}
Market based spectrum trading has been extensively studied to realize efficient spectrum utilization in cognitive radio networks (CRNs). In this paper, we utilize the concept of insurance in spectrum trading so as to improve spectrum efficiency in CRNs. We show that by additionally purchasing a specifically designed insurance contract from a PU, an SU can improve its utility since it will be insured against the potential accident, i.e., transmission failure incurred by excessively low SINR. Therefore insurance provides SUs more incentive to purchase PUs' channels and spectrum utilization in CRNs can be improved. In this paper, the original spectrum market including multiple PUs and multiple SUs are modeled as a hybrid market consisting of a spectrum market and an insurance market. In this hybrid market PUs serve as spectrum sellers as well as insurers and SUs act as spectrum buyers as well as insureds. We further model the hybrid market game as a four-stage Bayesian game between PUs and SUs. We characterize the second-best Pareto optimal (SBPO) market allocations and players' perfect Bayesian equilibrium (PBE) strategies. Furthermore, through extensive simulation, we have demonstrated that at the PBE, high risk and low risk SUs will respectively experience improvement in their utilities for approximately $23.5 \%$ and $4.6 \%$.
\end{abstract}

\section{INTRODUCTION}

Cognitive radio (CR) has been regarded as a promising paradigm to tackle the spectrum scarcity and inefficient spectrum usage in current wireless communication networks. CRNs are designed based on the notion of dynamic spectrum sharing where cognitive radio users have the capability to opportunistically share the radio spectrum.

Among mechanisms for dynamic spectrum sharing, marketdriven spectrum trading is one of the most commonly utilized frameworks. In [12], Niyato et al. analyzed the spectrum trading between multiple PUs and SUs. They modeled SUs' dynamic and evolutionary behavior as an evolutionary game and the competition among the PUs as a noncooperative game. In [3], Kim et al. studied the price and quality competitions of duopoly CRNs. The CRNs were modeled as a duopoly Wi-Fi 2.0 market in which two wireless service providers compete for customers and spectra. In [5] and [11], Duan et al. studied under spectrum supply uncertainty the optimal pricing and investment of a cognitive mobile virtual network operator (C-MVNO). In short-term, a C-MVNO can obtain spectrum opportunities dynamically by sensing and leasing the spectrum from the spectrum owner. This paper models the spectrum negotiation between C-MVNO and SUs as a Stackelberg game and analyze its equilibrium properties. Moreover, the issue of competitive pricing in CRNs was studied in [9] in which Niyato et al. modeled the spectrum trading between PUs and SUs as a Bertrand game and analyzed its Nash equilibrium.

Moreover, auction based spectrum trading has also been proved to be an effective framework for dynamic spectrum sharing and literatures abound in the realm of spectrum auction. In [14], Zhou et al. proposed a truthful and computationally-efficient spectrum auction mechanism, VERITAS. Zhou et al. in [6], proposed TRUST, the first solution enabling truthful double spectrum auctions. In [15], Jia et al. showed that the primary users' revenue can be maximized by introducing a specifically designed spectrum auction framework. In [10], Gao et al. proposed a multiauctioneer progressive auction framework enhancing spectrum efficiency in CRNs with multiple PUs and SUs. In [4] and [1], Gopinathan et al. respectively studied the revenue-maximizing and strategy-proof auction mechanism to achieve fair and efficient spectrum allocation. Furthermore, Deek et al. in [2] proposed Topaz, a truthful online spectrum auction design to deal with bid and time-based cheating in online auctions.

In addition, only recently, contract-based dynamic spectrum sharing has also been proved to be a promising mechanism for spectrum trading. In [8], Gao et al. characterized the incentive compatible and individually rational contractual forms for SUs to purchase PUs' spectra with the quality-price designed for their own types. Furthermore, in [7], Duan et al. modeled the $\mathrm{CRNs}$ as a labor market and incorporated contract theory to resolve the problem of cooperative relay between PUs and SUs. However, all the aforementioned spectrum trading mechanisms fail to consider the incentives for SUs to purchase PUs' channels in the scenario where SUs face potential transmission failures. Under such circumstances spectrum efficiency will thus be undermined in that SUs may not have enough incentives to purchase PUs' channels.

Therefore, in this paper, we seek to improve spectrum efficiency in CRNs by incorporating insurance mechanism into spectrum trading. Insurance theory has been widely studied in the realm of economy. In [16], Clarke studied the duty of good faith in the law of designing insurance contracts. Moreover, Schiller in [17] proposed that a semi-separating equilibrium may occur in insurance markets where misrepresentation takes the form of fraudulent claiming. In our framework, both PUs and SUs possess double identities that is PUs serve as spectrum sellers as well as insurers and SUs act as spectrum 
buyers as well as insureds. Facing the insurance contracts offered by PUs, any arbitrary SU determines whether to simply purchase a channel or meanwhile signs an insurance contract with the PU to obtain insurance for the potential accident, i.e., transmission failure incurred by the excessively low SINR. In this paper, we model the market game as a four-stage Bayesian game and characterize the second-best Perato optimal allocations (SBPO) and the perfect Bayesian equilibrium (PBE). Spectrum utilization is shown to be improved by introducing insurance mechanism into spectrum trading and both PUs and SUs will experience increase in their utilities at the PBE. The contributions of this paper are demonstrated as follows:

- We show that by utilizing insurance mechanism in spectrum trading spectrum efficiency in CRNs, where SUs face potential transmission failures, can be improved. Furthermore, we model the market game between PUs and SUs as a hybrid market and characterize the SBPO market allocations, the PBE contractual forms and players' PBE strategies. Moreover, we derive the conditions for the existence of the PBE.

- We analyze the information asymmetry between PUs and SUs about SUs' risk types and study the effect of PUs' stochastic risk verification on SUs' risk type cheating.

- Through extensive simulation, we have demonstrated that at equilibrium, both high risk and low risk SUs will respectively experience an improvement in their utilities for approximately $23.5 \%$ and $4.6 \%$.

The rest of this paper is organized as follows: in section II, we introduce the network model, hybrid market structure and elements of the PBE. In section III, we characterize the SBPO market allocations. In section IV, we derive the conditions for the existence of the PBE and characterize the PBE contractual forms and players' PBE strategies. In section V, we provide the numerical and simulation results and in section VI, we discuss the conclusions and the future work.

\section{Network Model, Hybrid Market Structure AND PBE ELEMENTS}

\section{A. Network Model}

We consider a CR network including one primary operator (PO), multiple PUs and multiple SUs. The PO can be an access point or a base station. Any $\mathrm{SU} i$ is a dedicated transmitter-receiver pair, $\mathrm{Tx}_{i}-\mathrm{Rx}_{i}$ and we denote the set of SUs as $\left.\left\{\mathrm{Tx}_{k}-\mathrm{Rx}_{k}\right\}\right|_{k=1} ^{n}$. We assume that $\mathrm{SU}$ transmitters always have packets to send to their dedicated receivers. Moreover, such transmission can be carried out only when an SU has successfully purchased a channel from a PU. The network model is demonstrated in Figure 1.

SUs in this network are heterogeneous in terms of their distances $d$ from the PO. Within any arbitrary time slot, we use $f(d)$ to denote the p.d.f. of the distance between an SU and the PO. The specific expression of $f(d)$ is determined by SUs' mobility model. For example, if SUs move according to the restricted mobility model in [13], $f(d)$ is exponentially decaying. Then, we define the type of an SU in Definition 1.

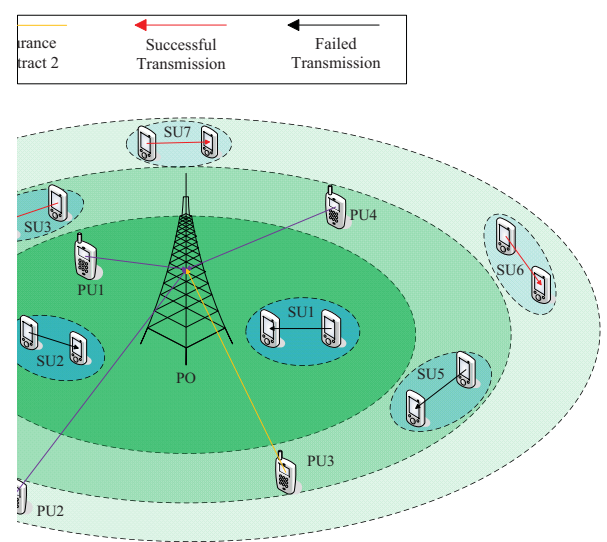

Fig. 1. Illustration of Network Model: SUs 1 and 2 belong to type-1, SUs 3,4 and 5 belong to type-2 and SUs 6 and 7 belong to type-3. Due to interference from the PO, transmission from SU Txes 1, 2 and 5 to their RXes fail. PUs 1, 2 and 4 propose insurance contract 1 and PUs 3 proposes insurance contract 2.

Definition 1. We draw $q$ concentric circles centered at the $\mathrm{PO}$ respectively with radiuses $r_{1}<\cdots<r_{q}$, and we call SU $i$ a type- $j \mathrm{SU}$ if, in the current time slot, SU $i$ locates in the $(j-1)$ th $(j \geq 2)$ annulus, i.e., $r_{j-1}<d_{i}<r_{j}$ and type-1 SU, if it is within the innermost circular region with $r_{n}$ being the PO's maximum interference distance. Hence, the probability for an SU to be a type- $j(j \geq 2) \mathrm{SU}$ is $\beta_{j}=\int_{r_{j-1}}^{r_{j}} f(x) d x$ and type- 1 SU $\beta_{1}=\int_{0}^{r_{j}} f(x) d x$.

The SINR at SU receiver $i$ with SU transmitter $i$ and SU receiver $i$ respectively locating at point $X_{t}^{i}$ and $X_{r}^{i}$ is:

$$
\gamma_{i}=\frac{P_{i} \kappa_{i}\left\|X_{t}^{i}-X_{r}^{i}\right\|^{-\alpha}}{I_{P O}^{i}+I_{P U}^{i}+\sigma_{i}^{2}}
$$

We assume that the transmission power of SU transmitter $i P_{i}$, the path loss factor $L_{i}\left(X_{t}, X_{r}\right)=\kappa_{i}\left\|X_{t}-X_{r}\right\|^{-\alpha}$, the noise variance $\sigma_{i}^{2}$ and $I_{P U}^{i}$ are approximately identical among all SUs. Hence, interference is primarily incurred by the PO. Therefore, the distance between SU $i$ and the PO differentiates the SUs in that $I_{P O}^{i}$ is determined by $d_{i}$. Furthermore, we assume that SUs of the same type have approximately the same SINR at their SU receivers. Furthermore, we assume that SUs in this network carry out packet-based transmission. When, for any arbitrary type- $j \mathrm{SU}, \gamma_{j}$ falls below a certain threshold $\gamma_{t h}$, the transmission from the SU transmitter to the SU receiver will fail because, in this scenario, the particular SU receiver will be unable to decode the packets transmitted by its transmitter due to excessively low SINR. Hence, the accident probability, i.e., the probability of failed transmission for any arbitrary type- $j$ SU can be denoted as $\xi_{j}=\phi\left(\gamma_{j}\right)$ satisfying that $\phi(\bullet)$ is strictly monotonically decreasing with range $(0,1)$. Apparently, we have $0<\xi_{j}<\xi_{j-1}<1(j \geq 2)$.

In this paper, we consider the scenario with acknowledgement of successful transmission that is if and only if an SU receiver successfully receives and decodes the packets sent by its dedicated transmitter, it sends a feedback signal, ACK 
to its transmitter. Thus, based on the presence or absence of the ACK signal, the particular SU transmitter will be able to decide whether the current transmission is successful. In this network, SU transmitters send identical number of packets denoted by $\mathcal{P}_{S}$ in the case of successful transmission.

The primary network consists of $m$ PUs and one PO. We assume that PUs always have idle spectra to trade with SUs and the PO serves as the interface of the spectrum trading and insurance contract signing between PUs and SUs. In this paper, we assume that the PO possesses the location information of SUs in the local area and charge $c$ for each consultation of this information issued by a PU. The analysis of the strategic behavior of the PO is beyond the scope of this paper.

\section{B. Hybrid Market Structure}

The hybrid market consists of a spectrum market and an insurance market. PUs and SUs in this market have double identities that is PUs serve as spectrum sellers as well as insurers and SUs act as spectrum buyers as well as insureds. Apart from the price of its channels $\tau_{i}$, any arbitrary PU $i$ offers a menu of insurance contracts w.r.t. different types of SUs i.e., $\mathcal{C}_{i}=\left(\mathcal{C}_{1}^{i}, \cdots, \mathcal{C}_{q}^{i}\right)$. We assume that all PUs sell channels at identical price $\tau_{i}=\tau, \forall i \in\{1, \cdots, m\}$. The channels sold by PUs expire after one time slot and PUs can resell these channels if they are still idle in the next time slot. SUs can purchase only one channel and sign one insurance contract at the beginnging of every time slot. In every time slot, enlightened by [17], [18] and [19] we model the market game as a four-stage Bayesian game.

- Stage I (Insurance contract proposing)

Each PU $i$ accesses the PO and proposes a menu of insurance contracts $\mathcal{C}^{i}=\left(\mathcal{C}_{1}^{i}, \cdots, \mathcal{C}_{q}^{i}\right)$.

- Stage II (Channel purchasing and contract signing) SUs access the PO to obtain the channel pricing and insurance contracts information. If an SU decides to sign an insurance contract, it meanwhile purchases a channel sold by the same PU. Otherwise, the SU stochastically purchases a channel from one of the PUs.

\section{- Stage III (Data transmission and claim filing)}

SUs carry out data transmission from SU transmitters to $\mathrm{SU}$ receivers. If the transmission fails, the SU then files a claim for indemnity to its insurer through the PO if it has signed an insurance contract with the PU in stage II.

\section{- Stage IV (Auditing and indemnity paying)}

When PU $i$ receives a claim for indemnity, it conducts risk verification of the $\mathrm{SU}$ with probability $p_{i} \in[0,1]$ by consulting the PO the location information of the SU. The PU can then determine whether this SU has cheated on its risk types and has selected an insurance contract designed for other SU types. If no risk type cheating is established, the PU pays the indemnity to the SU. Otherwise, it rescinds the contract and pays no indemnity.

In Definition 2, we define the form of insurance contracts.

Definition 2. An insurance contract $\mathcal{C}_{j}^{i}$ proposed by PU $i$ to type- $j$ SUs has the form of $\mathcal{C}_{j}^{i}=\left(\lambda_{j}^{i}, \theta_{j}^{i}\right)$ in which $\lambda_{j}^{i}$ represents the insurance premium, $\theta_{j}^{i}$ the net payoff in the case of an accident and therefore the total indemnity $\lambda_{j}^{i}+\theta_{j}^{i}$.

For simplification of analysis, in this paper, we dwell on the scenario where only two categories of SUs, namely type$h$ and type- $l$, respectively denoting SUs with high and low accident probability, exist in this network. We divide the region inside the PO interference circle into two parts i.e., the inner circular region, $\mathcal{A}_{1}=\left\{X \mid 0<\left\|X-X_{P O}\right\| \leq r_{t h}\right\}$ and the outer annulus, $\mathcal{A}_{2}=\left\{X \mid r_{t h} \leq\left\|X-X_{P O}\right\| \leq r_{\max }\right\}$, with $X$ denoting the position of any arbitrary point, $X_{P O}$ the position of the PO, $r_{t h}$ the threshold radius and $r_{\max }$ the PO's maximum interference distance. Since SUs in region $\mathcal{A}_{1}$ suffer from higher level of interference from the PO than those located in region $\mathcal{A}_{2}$, we denote the former type of SUs type$h$ SUs and the latter type- $l$ SUs, respectively with accident probability $\xi_{h}=\phi\left(\gamma_{h}\right)$ and $\xi_{l}=\phi\left(\gamma_{l}\right)$ satisfying that $0<$ $\xi_{l}<\xi_{h}<1$. Moreover, the probability for an SU to be type- $h$ and type- $l$ are respectively $\beta_{h}=\iint_{\mathcal{A}_{1}} f(x)=\int_{0}^{r_{t h}} f(x) d x$ and $\beta_{l}=\iint_{\mathcal{A}_{2}} f(x)=\int_{r_{t h}}^{r_{\text {max }}} f(x) d x$. Hence, approximately we have $\beta_{h}+\beta_{l}=1$.

In this paper, we consider an insurance market with information asymmetry and costly risk verification. SUs have private knowledge of their risk types that are not available to PUs unless they carry out risk verification. PUs are charged for $c$ by the PO for each risk verification. It is necessary for PUs to verify SUs' risk levels because in insurance markets one gist of insurance contract design is to charge more premium for insureds with higher risk. Hence, type- $h$ SUs have incentive to misrepresent their risk types and sign the contracts designed for type- $l$ insureds to pay less insurance premium. For example, when SUs are offered actuarially fair full insurance contracts i.e., $\overline{\mathcal{C}}_{h}=\left\{\bar{\lambda}_{h}, \bar{\theta}_{h}\right\}=\left\{\xi_{h} L, L-\xi_{h} L\right\}$ for type$h$ SUs and $\overline{\mathcal{C}}_{l}=\left\{\bar{\lambda}_{l}, \bar{\theta}_{l}\right\}=\left\{\xi_{l} L, L-\xi_{l} L\right\}$ for type-l SUs, with $L$ denoting an SU's loss incurred by an accident, since $\xi_{l} L<\xi_{h} L$, it is possible that a type- $h \mathrm{SU}$ chooses $\overline{\mathcal{C}}_{l}$. For the same reason, PUs only carry out risk verification for alleged type- $l$ claimants. For the costly nature of audition, there is an inherent trade-off between the avoidance of paying indemnity with the payment of audition cost. Thus, each PU $i$ audits stochastically type- $l$ claimant with probability $p_{i} \in[0,1]$.

\section{Elements of the four-stage Bayesian game}

In this subsection, we define the basic elements of this four-stage Bayesian game including players' strategies, beliefs, utility functions and PBE conditions. In Definitions 3 and 4, we respectively define players' strategies and beliefs.

Definition 3. PUs' strategy profile is defined as $\{\mathbf{C}, \mathbf{p}(\cdot)\}$ with $\mathbf{C}=\left(\mathcal{C}_{1}, \cdots, \mathcal{C}_{m}\right)$ and $\mathbf{p}(\cdot)=\left(p_{1}(\cdot), \cdots, p_{m}(\cdot)\right)$ where $\mathcal{C}_{i}=\left(\mathcal{C}_{h}^{i}, \mathcal{C}_{l}^{i}\right)$ is the contract menu proposed by PU $i$ and $p_{i}(\mathbf{C})$ is its audition probability given contract profile $\mathbf{C}$. Moreover, the strategy of a type- $k$ SU is defined as $\pi_{k}(\cdot)=$ $\left(\pi_{k h}^{0}(\cdot), \pi_{k l}^{0}(\cdot), \cdots, \pi_{k h}^{m}(\cdot), \pi_{k l}^{m}(\cdot)\right)$ in which $\pi_{k j}^{i}(\mathbf{C})$ denotes the probability that given $\mathbf{C}$, a type- $k$ SU signs insurance contract $\mathcal{C}_{j}^{i}$ if $i \geq 1$ and signs no insurance contract if $i=0$ such that $\sum_{i=0}^{m}\left(\pi_{k h}^{i}+\pi_{k l}^{i}\right)=1$ and $k, j \in\{h, l\}$. Therefore, the overall strategy profile is $\left\{\mathbf{C}, \mathbf{p}(\cdot), \pi_{h}(\cdot), \pi_{l}(\cdot)\right\}$. 
Definition 4. The belief profile is denoted as $\eta(\cdot)=$ $\left(\eta_{1}(\cdot), \cdots, \eta_{m}(\cdot)\right)$ in which $\eta_{i}(\mathbf{C})$ is the probability in PU $i$ 's belief that a $\mathcal{C}_{l}^{i}$ clamant is in fact type- $h$ given the contract profile $\mathbf{C}$. Moreover, $\eta_{i}(\mathbf{C})$ is calculated through Bayes law:

$$
\begin{aligned}
& \eta_{i}(\mathbf{C})=\frac{\beta_{h} \xi_{h} \pi_{h l}^{i}(\mathbf{C})}{\beta_{h} \xi_{h} \pi_{h l}^{i}(\mathbf{C})+\left(1-\beta_{h}\right) \xi_{l} \pi_{l l}^{i}(\mathbf{C})}, \\
& \text { s.t. } \pi_{h l}^{i}(\mathbf{C})+\pi_{l l}^{i}(\mathbf{C})>0, \forall i \in\{1, \cdots, m\}
\end{aligned}
$$

In definition 5 and 6 , we define players' utility functions.

Definition 5. (3)-(7) hold for $\forall i \in\{0, \cdots, m\}$.

- The expected utility of an SU that purchases an insurance contract designed for its own type is:

$$
\begin{aligned}
& R_{k k}\left(\mathcal{C}_{k}^{i}\right) \\
= & \left(1-\xi_{k}\right) r\left(U_{S}-\tau-\lambda_{k}^{i}\right)+\xi_{k} r\left(U_{F}-\tau+\theta_{k}^{i}\right), \\
& \text { s.t. } k \in\{h, l\}
\end{aligned}
$$

- The expected utility of a type- $h \mathrm{SU}$ that purchases $\mathcal{C}_{l}^{i}$ is:

$$
\begin{aligned}
& R_{h l}\left(\mathcal{C}_{l}^{i}, p_{i}\right) \\
= & \xi_{h}\left(\left(1-p_{i}\right) r\left(U_{S}-\tau+\theta_{l}^{i}\right)+p_{i} r\left(U_{F}-\tau\right)\right)+ \\
& \left(1-\xi_{h}\right) r\left(U_{S}-\tau-\lambda_{l}^{i}\right)
\end{aligned}
$$

- The expected utility of a type-l $\mathrm{SU}$ that purchases $\mathcal{C}_{h}^{i}$ is:

$$
\begin{aligned}
& R_{l h}\left(\mathcal{C}_{h}^{i}\right) \\
= & \left(1-\xi_{l}\right) r\left(U_{S}-\tau-\lambda_{h}^{i}\right)+\xi_{l} r\left(U_{F}-\tau+\theta_{h}^{i}\right)
\end{aligned}
$$

- The expected utility of a type-l $\mathrm{SU}$ is:

$$
\begin{aligned}
& \Lambda_{l}\left(\mathbf{C}, \pi_{l}(\cdot)\right) \\
= & \sum_{i=0}^{m}\left(\pi_{l h}^{i}(\mathbf{C}) R_{l h}\left(\mathcal{C}_{h}^{i}\right)+\pi_{l l}^{i}(\mathbf{C}) R_{l l}\left(\mathcal{C}_{l}^{i}\right)\right)
\end{aligned}
$$

- The expected utility of a type- $h \mathrm{SU}$ is:

$$
\begin{aligned}
& \Lambda_{h}\left(\mathbf{C}, \mathbf{p}(\cdot), \pi_{h}(\cdot)\right) \\
= & \sum_{i=0}^{m}\left(\pi_{h h}^{i}(\mathbf{C}) R_{h h}\left(\mathcal{C}_{h}^{i}\right)+\pi_{h l}^{i}(\mathbf{C}) R_{h l}\left(\mathcal{C}_{l}^{i}, p_{i}(\mathbf{C})\right)\right)
\end{aligned}
$$

In (3)-(5), $U_{S}=\mathcal{P}_{S} \Delta$ denotes the successful transmission reward which we assume is linearly related with $\mathcal{P}_{S}$, the number of packets received by the SU receiver and $U_{F}$ denotes the transmission reward in the case of an accident which is approximately 0 . Hence, an SU's loss incurred by an accident is $L=\left(U_{S}-\tau\right)-\left(U_{F}-\tau\right)$. Moreover, $r(\bullet)$ satisfies that $r^{\prime}(\bullet)>0$ and $r^{\prime \prime}(\bullet)<0$ and $\mathcal{C}_{h}^{0}=\mathcal{C}_{l}^{0}=(0,0)$ represent the case in which the SU remains uninsured.

Definition 6. (8)-(12) hold for $\forall i \in\{1, \cdots, m\}$.

- The expected profit of PU $i$ for contract $\mathcal{C}_{h}^{i}$ is:

$$
G_{h k}\left(\mathcal{C}_{h}^{i}\right)=\left(1-\xi_{k}\right) \lambda_{h}^{i}-\xi_{k} \theta_{h}^{i}, k \in\{h, l\}
$$

- PU $i$ 's expected profit for $\mathcal{C}_{l}^{i}$ signed by a type-l $\mathrm{SU}$ is:

$$
G_{l l}\left(\mathcal{C}_{l}^{i}, p_{i}\right)=\left(1-\xi_{l}\right) \lambda_{l}^{i}-\xi_{l}\left(\theta_{l}^{i}+p_{i} c\right)
$$

- PU $i$ 's expected profit for $\mathcal{C}_{l}^{i}$ signed by a type- $h \mathrm{SU}$ is:

$$
G_{l h}\left(\mathcal{C}_{l}^{i}, p_{i}\right)=\left(1-\xi_{h}\right) \lambda_{l}^{i}-\xi_{h}\left(\left(1-p_{i}\right) \theta_{l}^{i}+p_{i} c\right)
$$

- PU $i$ 's expected utility from an insurance contract is:

$$
\begin{aligned}
& \Phi_{i}\left(\mathbf{C}, \mathbf{p}(\cdot), \pi_{h}(\cdot), \pi_{l}(\cdot)\right) \\
= & \beta_{h}\left(\pi_{h h}^{i}(\mathbf{C}) G_{h h}\left(\mathcal{C}_{h}^{i}\right)+\pi_{h l}^{i}(\mathbf{C}) G_{l h}\left(\mathcal{C}_{l}^{i}, p_{i}(\mathbf{C})\right)\right)+ \\
& \beta_{l}\left(\pi_{l h}^{i}(\mathbf{C}) G_{h l}\left(\mathcal{C}_{h}^{i}\right)+\pi_{l l}^{i}(\mathbf{C}) G_{l l}\left(\mathcal{C}_{l}^{i}, p_{i}(\mathbf{C})\right)\right)
\end{aligned}
$$

- PU $i$ 's expected utility given that it has sold $M$ channels and $N$ insurance contracts such that $M \geq N$ is:

$$
\begin{aligned}
& \Omega_{i}\left(\mathbf{C}, \mathbf{p}(\cdot), \pi_{h}(\cdot), \pi_{l}(\cdot), M, N\right) \\
= & M \tau+N \Phi_{i}\left(\mathbf{C}, \mathbf{p}(\cdot), \pi_{h}(\cdot), \pi_{l}(\cdot)\right)
\end{aligned}
$$

The rest of this paper seeks to characterize the equilibrium of this four-stage Bayesian game. Such an equilibrium is a perfect Bayesian equilibrium formally defined in Definition 7.

Definition 7. $\mathcal{E}=\left\{\mathbf{C}^{*}, \mathbf{p}^{*}(\cdot), \pi_{h}^{*}(\cdot), \pi_{l}^{*}(\cdot), \eta_{i}^{*}(\cdot)\right\}$ is a perfect Bayesian equilibrium of the four-stage Bayesian game if it satisfies:

$$
\begin{aligned}
\Lambda_{h}\left(\mathbf{C}, \mathbf{p}^{*}(\cdot), \pi_{h}(\cdot)\right) & \leq \Lambda_{h}\left(\mathbf{C}, \mathbf{p}^{*}(\cdot), \pi_{h}^{*}(\cdot)\right), \forall \pi_{h}(\cdot), \mathbf{C} \\
\Lambda_{l}\left(\mathbf{C}, \pi_{l}(\cdot)\right) & \leq \Lambda_{l}\left(\mathbf{C}, \pi_{l}^{*}(\cdot)\right), \forall \pi_{l}(\cdot), \mathbf{C}
\end{aligned}
$$

The following (15)-(17) hold for $\forall i \in\{1, \cdots, m\}$ :

$$
\begin{aligned}
p_{i}(\mathbf{C})\left(\eta_{i}^{*}(\mathbf{C}) \theta_{l}^{i}-c\right) \leq p_{i}^{*}(\mathbf{C})\left(\eta_{i}^{*}(\mathbf{C}) \theta_{l}^{i}-c\right), \forall p_{i}(\cdot), \mathbf{C} \\
\quad \Omega_{i}\left(\left(\mathcal{C}_{i}, \mathbf{C}_{-i}^{*}\right), \mathbf{p}^{*}(\cdot), \pi_{h}^{*}(\cdot), \pi_{l}^{*}(\cdot), M, N\right) \\
\leq \Omega_{i}\left(\mathbf{C}^{*}, \mathbf{p}^{*}(\cdot), \pi_{h}^{*}(\cdot), \pi_{l}^{*}(\cdot), M, N\right), \forall \mathcal{C}_{i}=\left(\mathcal{C}_{h}^{i}, \mathcal{C}_{l}^{i}\right) \\
\eta_{i}^{*}(\mathbf{C})=\frac{\beta_{h} \xi_{h} \pi_{h l}^{i *}(\mathbf{C})}{\beta_{h} \xi_{h} \pi_{h l}^{i *}(\mathbf{C})+\left(1-\beta_{h}\right) \xi_{l} \pi_{l l}^{i *}(\mathbf{C})}, \quad \forall \mathbf{C} .
\end{aligned}
$$

(13) holds for $\forall \pi_{h}(\cdot)$ and $\forall \mathbf{C}$ conveying that $\pi_{h}^{*}(\cdot)$ is the optimal contract choice for type- $h$ SUs given PUs' auditing strategies $\mathbf{p}^{*}(\cdot)$. Similarly, holding for $\forall \pi_{l}(\cdot)$ and $\forall \mathbf{C}$, (14) reveals the optimality of $\pi_{l}^{*}(\cdot)$ among type-l SUs' contract selection strategies. (15) means for $\forall \mathbf{C}, p_{i}^{*}(\cdot)$ is the optimal audition strategy for PU $i$ given its belief $\eta_{i}^{*}(\mathbf{C})$. (16) says given other PUs' contract profile $\mathbf{C}_{-i}^{*}=\left(\mathcal{C}_{1}^{*}, \cdots, \mathcal{C}_{i-1}^{*}, \mathcal{C}_{i+1}^{*}, \cdots, \mathcal{C}_{m}^{*}\right)$, $\mathcal{C}_{i}^{*}$ is the optimal contract offer for PU $i$ and (17) states that $\eta^{*}(\cdot)$ can be calculated from $\pi_{h}^{*}(\cdot)$ and $\pi_{l}^{*}(\cdot)$ by Bayesian law. Hence, the PBE strategy profile $\left\{\mathbf{C}^{*}, \mathbf{p}^{*}(\cdot), \pi_{h}^{*}(\cdot), \pi_{l}^{*}(\cdot)\right\}$ satisfies sequential rationality given $\eta^{*}(\cdot)$.

\section{Characterization of Second-best Pareto OPTIMAL (SBPO) AlLOCATIONS}

In this section, we characterize the PBE of this four-stage Bayesian game by delving into its SBPO allocations. For any strategy profile, $\left\{\mathbf{C}, \mathbf{p}(\cdot), \pi_{h}(\cdot), \pi_{l}(\cdot)\right\}$, a market allocation is defined as $\left\{\mathbf{C}, \mathbf{p}, \pi_{h}, \pi_{l}\right\}$ with $\mathbf{p}=\mathbf{p}(\mathbf{C}), \pi_{h}=\pi_{h}(\mathbf{C})$ and $\pi_{l}=\pi_{l}(\mathbf{C})$. In this paper, we define Pareto optimality in second-best sense because of the constraints introduced in $\mathbb{C}_{1}$ later this section. Then, we introduce Lemmas 1 and 2 that shed light upon several basic properties of the PBE.

Lemma 1. For PBE $\mathcal{E}=\left\{\mathbf{C}^{*}, \mathbf{p}^{*}(\cdot), \pi_{h}^{*}(\cdot), \pi_{l}^{*}(\cdot), \eta_{i}^{*}(\cdot)\right\}$, such that $\pi_{h l}^{i *}(\mathbf{C})+\pi_{l l}^{i *}(\mathbf{C})>0, \forall \mathbf{C}$, we have $p_{i}^{*}(\mathbf{C})<1$ and $p_{i}^{*}(\mathbf{C})\left(\eta_{i}^{*}(\mathbf{C}) \theta_{l}^{i}-c\right)=0$. Furthermore, if $\pi_{h l}^{i *}(\mathbf{C})=0$ and $\pi_{l l}^{i *}(\mathbf{C})>0$, then we have $p_{i}^{*}(\mathbf{C})=0$. 
Proof: Suppose that $p_{i}^{*}(\mathbf{C})=1$, we have

$$
\begin{aligned}
& R_{h l}\left(\mathcal{C}_{l}^{i}, 1\right) \\
= & \xi_{h} r\left(U_{F}-\tau\right)+\left(1-\xi_{h}\right) r\left(U_{S}-\tau-\lambda_{l}^{i}\right) \\
< & \xi_{h} r\left(U_{F}-\tau\right)+\left(1-\xi_{h}\right) r\left(U_{S}-\tau\right) \\
= & R_{h h}\left(\mathcal{C}_{h}^{0}\right)
\end{aligned}
$$

Hence, we also have $\pi_{h l}^{i *}=0$. Then (17) gives $\eta_{i}^{*}(\mathbf{C})=0$ and $\eta_{i}^{*}(\mathbf{C}) \theta_{l}^{i}=0<c$. Therefore, $p_{i}^{*}(\mathbf{C})=0$ according to (15), which contradicts with the assumption that $p_{i}^{*}(\mathbf{C})=1$. Thus, $p_{i}^{*}(\mathbf{C})<1$. From (15), we have $p_{i}^{*}(\mathbf{C})\left(\eta_{i}^{*}(\mathbf{C}) \theta_{l}^{i}-c\right)=0$. Specifically, for the case that $\pi_{h l}^{i *}(\mathbf{C})=0$ and $\pi_{l l}^{i *}(\mathbf{C})>0$, we have $\eta_{i}^{*}(\mathbf{C})=0$ and $p_{i}^{*}(\mathbf{C})=0$ from (15) and (17).

Remarks. Lemma 1 conveys that every PU carries out stochastic audition at PBE i.e., $p_{i}^{*}(\mathbf{C})<1$. This is somewhat intuitive because when risk verification is carried out by PU $i$ with probability 1 , type- $h$ SUs will be better off to remain uninsured or sign insurance contracts other than $\mathcal{C}_{l}^{i}$. Furthermore, this in turn indicates that audition is unnecessary, i.e., $p_{i}^{*}(\mathbf{C})=0$. Moreover, audition is conducted with positive probability only when $\eta_{i}^{*}(\mathbf{C})$ reaches a threshold $\frac{c}{\theta_{i}^{i}}$.

Lemma 2. The profit of insurance contract $\mathcal{C}_{l}^{i}$ for any PU $i$ at $\operatorname{PBE} \mathcal{E}=\left\{\mathbf{C}^{*}, \mathbf{p}^{*}(\cdot), \pi_{h}^{*}(\cdot), \pi_{l}^{*}(\cdot), \eta^{*}(\cdot)\right\}$ can be represented by SUs' contract choices $\pi_{h l}^{i *}$ and $\pi_{l l}^{i *}$ without explicitly incorporating PU $i$ 's audition probability $p_{i}^{*}(\mathbf{C})$.

Proof: From Lemma 1, we know that $p_{i}^{*}(\mathbf{C})\left(\eta_{i}^{*}(\mathbf{C}) \theta_{l}^{i}-\right.$ $c)=0, p_{i}^{*}(\mathbf{C})<1$ and PUs are indifferent about whether to carry out risk verification or not. Hence, we have

$$
\begin{aligned}
& \beta_{h} \pi_{h l}^{i *}(\mathbf{C}) G_{l h}\left(\mathcal{C}_{l}^{i}, p_{i}^{*}(\mathbf{C})\right)+\beta_{l} \pi_{l l}^{i *} G_{l l}\left(\mathcal{C}_{l}^{i}, p_{i}^{*}(\mathbf{C})\right) \\
= & \beta_{h} \pi_{h l}^{i *}(\mathbf{C}) G_{l h}\left(\mathcal{C}_{l}^{i}, 0\right)+\beta_{l} \pi_{l l}^{i *} G_{l l}\left(\mathcal{C}_{l}^{i}, 0\right) \\
= & \beta_{h} \pi_{h l}^{i *}(\mathbf{C}) G_{l h}^{0}\left(\mathcal{C}_{l}^{i}\right)+\beta_{l} \pi_{l l}^{i *} G_{l l}^{0}\left(\mathcal{C}_{l}^{i}\right)
\end{aligned}
$$

Therefore, we have proved Lemma 2.

Then, in $\mathbb{C}_{1}$ we introduce the constraints that SBPO allocations have to satisfy in this Bayesian game. Such constraints include information asymmetry between SUs and PUs about SUs' risk types shown in (21) and (22) and no pre-commitment of PUs' audition strategies demonstrated in (18) and (19). Furthermore, (20) requires that the expected profit that PU $i$ can get by proposing an insurance contract should be no smaller than $\omega_{i}$, because otherwise PUs will have no incentive to propose insurance contracts. If an allocation $\left\{\mathbf{C}, \mathbf{p}, \pi_{h}, \pi_{l}\right\}$ satisfies constraint $\mathbb{C}_{1}$, we call it a feasible allocation.

$\mathbb{C}_{1}$ : A feasible allocation $\left\{\mathbf{C}, \mathbf{p}, \pi_{h}, \pi_{l}\right\}$ should satisfy the following constraints for $\forall i \in\{1, \cdots, m\}$ and $\forall \mathbf{C}$.

$$
\begin{aligned}
& \left(\beta_{h} \xi_{h} \pi_{h l}^{i}+\left(1-\beta_{h}\right) \xi_{l} \pi_{l l}^{i}\right) c=\beta_{h} \xi_{h} \pi_{h l}^{i} \theta_{l}^{i}, \text { if } p_{i}>0 \\
& \left(\beta_{h} \xi_{h} \pi_{h l}^{i}+\left(1-\beta_{h}\right) \xi_{l} \pi_{l l}^{i}\right) c \geq \beta_{h} \xi_{h} \pi_{h l}^{i} \theta_{l}^{i}, \text { if } p_{i}=0 \\
& \Phi_{i}\left(\mathbf{C}, \pi_{h}, \pi_{l}\right) \\
= & \left(1-\beta_{h}\right)\left(\pi_{l h}^{i}(\mathbf{C}) G_{h l}\left(\mathcal{C}_{h}^{i}\right)+\pi_{l l}^{i}(\mathbf{C}) G_{l l}^{0}\left(\mathcal{C}_{l}^{i}\right)\right)+ \\
& \beta_{h}\left(\pi_{h h}^{i}(\mathbf{C}) G_{h h}\left(\mathcal{C}_{h}^{i}\right)+\pi_{h l}^{i}(\mathbf{C}) G_{l h}^{0}\left(\mathcal{C}_{l}^{i}\right)\right) \geq \omega_{i} \\
& \Lambda_{h}\left(\mathbf{C}, \mathbf{p}, \pi_{h}\right) \\
= & \max \left\{R_{h h}\left(\mathcal{C}_{h}^{i}\right), R_{h l}\left(\mathcal{C}_{l}^{i}, p_{i}\right) \mid \forall i \in\{1, \cdots, m\}\right\}
\end{aligned}
$$

$$
\begin{aligned}
& \Lambda_{l}\left(\mathbf{C}, \mathbf{p}, \pi_{l}\right) \\
= & \max \left\{R_{l h}\left(\mathcal{C}_{h}^{i}\right), R_{l l}\left(\mathcal{C}_{l}^{i}\right) \mid \forall i \in\{1, \cdots, m\}\right\}
\end{aligned}
$$

Then in Definition 8, we define SBPO market allocations.

Definition 8. An allocation $\left\{\mathbf{C}, \mathbf{p}, \pi_{h}, \pi_{l}\right\}$ that satisfies $\mathbb{C}_{1}$ is an SBPO allocation if no other feasible allocation $\left\{\mathbf{C}^{\prime}, \mathbf{p}^{\prime}, \pi_{h}^{\prime}, \pi_{l}^{\prime}\right\}$ exists satisfying that:

$$
\Lambda_{k}\left(\mathbf{C}^{\prime}, \mathbf{p}^{\prime}, \pi_{k}^{\prime}\right) \geq \Lambda_{k}\left(\mathbf{C}, \mathbf{p}, \pi_{k}\right), k \in\{h, l\}
$$

In the following analysis, we characterize SBPO allocations by considering a series of constrained optimization problems from $\mathbb{O}_{1}$ to $\mathbb{O}_{3}$. Firstly, we consider problem $\mathbb{O}_{1}$.

$$
\begin{aligned}
& \mathbb{O}_{1}\left(\beta_{h}, c, \nu_{0}, \omega_{1}, \cdots, \omega_{m}\right): \\
& \quad\left\{\widehat{\mathbf{C}}, \widehat{\mathbf{p}}, \widehat{\pi}_{h}, \widehat{\pi}_{l}\right\}=\arg \max \Lambda_{l}\left(\mathbf{C}, \pi_{l}\right), \\
& \text { s.t. } \mathbb{C}_{1}\left(\beta_{h}, c, \omega_{1}, \cdots, \omega_{m}\right) \text { and } \Lambda_{h}\left(\widehat{\mathbf{C}}, \widehat{\mathbf{p}}, \widehat{\pi}_{h}\right) \geq \nu_{0}
\end{aligned}
$$

The solution of $\mathbb{O}_{1}\left(\beta_{h}, c, \nu_{0}, \omega_{1}, \cdots, \omega_{m}\right)$ sheds light upon the characterizations of the SBPO allocations of the four-stage Bayesian game because any SBPO allocation $\left\{\mathbf{C}, \mathbf{p}, \pi_{h}, \pi_{l}\right\}$ is in fact an optimal solution to $\mathbb{O}_{1}\left(\beta_{h}, c, \nu_{0}, 0, \cdots, 0\right)$ satisfying that $\nu_{0}=\sum_{i=1}^{m}\left(\pi_{h h}^{i}(\mathbf{C}) R_{h h}\left(\mathcal{C}_{h}^{i}\right)+\pi_{h l}^{i}(\mathbf{C}) R_{h l}\left(\mathcal{C}_{l}^{i}, p_{i}(\mathbf{C})\right)\right)$.

To analyze $\mathbb{O}_{1}$, firstly we study the symmetric variant of $\mathbb{O}_{1}$ i.e., $\mathbb{O}_{2}\left(\beta_{h}, c, \nu_{0}, \omega_{0}\right)=\left.\mathbb{O}_{1}\left(\beta_{h}, c, \nu_{0}, \omega_{1}, \cdots, \omega_{m}\right)\right|_{\omega_{i}=\omega_{0}} ^{1 \leq m}$. In $\mathbb{O}_{2}$ PUs offer the same contract menu i.e., $\mathcal{C}=\left(\mathcal{C}_{l}, \mathcal{C}_{h}\right)$ and audit SUs' risk types with the same auditing probability. Moreover, SUs are evenly distributed among PUs. Then, in Theorems 1-4, we provide our results about the characterizations of the SBPO allocations of this four-stage Bayesian game. We use $\left\{\dot{\mathcal{C}}, \dot{p}, \dot{\pi}_{h}, \dot{\pi}_{l}\right\}$ to denote the optimal solution for $\mathbb{O}_{2}\left(\beta_{h}, c, \nu_{0}, \omega_{0}\right)$. In this paper, we restrict our analysis on the case where $\dot{p}>0$. Similar conclusions exist when $\dot{p}=0$.

Theorem 1. For $\left\{\dot{\mathcal{C}}, \dot{p}, \dot{\pi}_{h}, \dot{\pi}_{l}\right\}$ such that $\dot{p}>0$, SUs' contract selection strategy satisfies $\dot{\pi}_{l l}=1, \dot{\pi}_{l h}=0, \dot{\pi}_{h h}=1-\dot{\pi}_{h l}$ and (25),

$$
\dot{\pi}_{h l}=B\left(\dot{\theta}_{l}, \beta_{h}, c\right)=\frac{\left(1-\beta_{h}\right) \xi_{l} c}{\beta_{h} \xi_{h}\left(\dot{\theta}_{l}-c\right)} \in(0,1)
$$

Proof: We denote the value function of the constrained maximization problem $\mathbb{O}_{2}\left(\beta_{h}, c, \nu_{0}, \omega_{0}\right)$ as $\psi_{2}\left(\beta_{h}, c, \nu_{0}, \omega_{0}\right)$ and we denote $\mathbb{O}_{2}\left(\beta_{h}, c, \nu_{0}, \omega_{0}\right)$ under scenarios such that $\dot{p}>0$ and $\dot{p}=0$ as $\mathbb{O}_{2}^{\prime}\left(\beta_{h}, c, \nu_{0}, \omega_{0}\right)$ and $\mathbb{O}_{2}{ }^{\prime \prime}\left(\beta_{h}, c, \nu_{0}, \omega_{0}\right)$ respectively with value function $\psi_{2}^{\prime}\left(\beta_{h}, c, \nu_{0}, \omega_{0}\right)$ and $\psi_{2}^{\prime \prime}\left(\beta_{h}, c, \nu_{0}, \omega_{0}\right)$. Therefore, we have $\psi_{2}\left(\beta_{h}, c, \nu_{0}, \omega_{0}\right)=$ $\max \left\{\psi_{2}^{\prime}\left(\beta_{h}, c, \nu_{0}, \omega_{0}\right), \psi_{2}^{\prime \prime}\left(\beta_{h}, c, \nu_{0}, \omega_{0}\right)\right\}$. If for a feasible allocation $\mathcal{Q}, \dot{\pi}_{l l}<1$, there is another feasible allocation $\mathcal{Q}^{\prime}$ of $\mathbb{O}_{2}^{\prime \prime}\left(\beta_{h}, c, \nu_{0}, \omega_{0}\right)$ such that a type-l SU earns higher utility for allocation $\mathcal{Q}^{\prime}$ than $\mathcal{Q}$. Then, we consider allocations feasible in $\mathbb{O}_{2}{ }^{\prime \prime}\left(\beta_{h}, c, \nu_{0}, \omega_{0}\right)$ with no counterparts in $\mathcal{Q}$ that makes type-l SUs better off. Hence, we have $\dot{\pi}_{l l}=1$ and $\dot{\pi}_{l h}=0$.

Since $\dot{p}>0$, (18) holds and by some manipulation we can arrive at the conclusion (25).

Theorem 2. For $\left\{\dot{\mathcal{C}}, \dot{p}, \dot{\pi}_{h}, \dot{\pi}_{l}\right\}$ such that $\dot{p}>0$, type- $h$ SUs' minimum utility $\nu_{0}$ is less than the utility granted by the pooling contract $\mathcal{C}_{0}=\left(\bar{\xi} L+\omega_{0},(1-\bar{\xi}) L-\omega_{0}\right)$, with $\bar{\xi}=\beta_{h} \xi_{h}+\left(1-\beta_{h}\right) \xi_{l}$ and $\bar{\nu}_{0}=r\left(U_{S}-\bar{\xi}-\omega_{0}\right)$, that is

$$
R_{h h}\left(\dot{\mathcal{C}}_{h}\right)=R_{h l}\left(\dot{\mathcal{C}}_{l}, \dot{p}\right)=\nu_{0}<\bar{\nu}_{0}
$$


Proof: Since Theorem 1 reveals that $\dot{\mathcal{C}}_{l}$ is better off for type-l SU than $\dot{\mathcal{C}}_{h}$, then we have (27),

$$
R_{l l}\left(\dot{\mathcal{C}}_{l}\right) \geq R_{l h}\left(\dot{\mathcal{C}}_{h}\right)
$$

Since, $\dot{\pi}_{l l}<1$ and $\dot{\pi}_{h l}>0$, we have (28),

$$
R_{h l}^{\prime}\left(\dot{\mathcal{C}}_{l}\right)=R_{h l}\left(\dot{\mathcal{C}}_{l}, 0\right)>R_{h h}\left(\dot{\mathcal{C}}_{h}\right)
$$

Moreover, without loss of generality, we also assume (29),

$$
R_{h h}\left(\dot{\mathcal{C}}_{h}\right) \geq \nu_{0}
$$

Suppose that $\nu_{0} \geq \bar{\nu}_{0}$, we have $\dot{\pi}_{h h} R_{h h}\left(\dot{\mathcal{C}}_{h}\right)+\dot{\pi}_{h l} G_{l h}^{0}\left(\dot{\mathcal{C}}_{l}\right)<$ $\left(\bar{\xi}-\xi_{h}\right) L+\omega_{0}$. From (20), we have $G_{l l}^{0}\left(\dot{\mathcal{C}}_{l}\right)>\left(\bar{\xi}-\xi_{l}\right) L+$ $\omega_{0}$ and $R_{l l}\left(\dot{\mathcal{C}}_{l}\right)<\bar{\nu}_{0}<R_{h l}\left(\dot{\mathcal{C}}_{l}, 0\right)$. Then we construct an allocation $\mathcal{Q}^{1}=\left\{\mathcal{C}_{h}^{1}, \mathcal{C}_{l}^{1}, p^{1}, \pi_{h h}^{1}, \pi_{h l}^{1}, \pi_{l h}^{1}, \pi_{l l}^{1}\right\}$ with $p^{1}=0$, $\pi_{h h}^{1}=1, \pi_{h l}^{1}=0, \pi_{l h}^{1}=0, \pi_{l l}^{1}=1$, and $\left(\mathcal{C}_{h}^{1}, \mathcal{C}_{l}^{1}\right)=\left(\mathcal{C}_{h}^{\prime}, \mathcal{C}_{l}^{\prime}\right)$. On one hand contract $\mathcal{C}_{l}^{\prime}$ satisfies $\lambda_{l}^{\prime}+\theta_{l}^{\prime}=L$ and $R_{l l}\left(\mathcal{C}_{l}^{\prime}\right)=$ $R_{l l}\left(\dot{\mathcal{C}}_{l}\right)$ which reveals that $G_{l l}^{0}\left(\mathcal{C}_{l}^{\prime}\right)>G_{l l}^{0}\left(\dot{\mathcal{C}}_{l}\right)$ and $R_{h l}^{\prime}\left(\dot{\mathcal{C}}_{l}\right)>$ $R_{h l}^{\prime}\left(\mathcal{C}_{l}^{\prime}\right)$. On the other hand, contract $\mathcal{C}_{h}^{\prime}$ satisfies that $\mathcal{C}_{h}^{\prime}=\dot{\mathcal{C}}_{h}$ when $\dot{\pi}_{h h}>0$ and $G_{h h}\left(\dot{\mathcal{C}}_{h}\right) \leq G_{l h}^{0}\left(\dot{\mathcal{C}}_{l}\right)$ and $\mathcal{C}_{h}^{\prime}=\dot{\mathcal{C}}_{l}$ when $\dot{\pi}_{h h}=0$. Therefore, we have $R_{l l}\left(\mathcal{C}_{l}^{\prime}\right) \geq R_{l l}\left(\mathcal{C}_{h}^{\prime}\right), R_{h h}\left(\mathcal{C}_{h}^{\prime}\right)>$ $R_{h l}^{\prime}\left(\mathcal{C}_{l}^{\prime}\right)$ and $R_{h h}\left(\mathcal{C}_{h}^{\prime}\right)>\nu_{0}$. Then from (20), we have

$$
\begin{aligned}
& \beta_{h} G_{h h}\left(\mathcal{C}_{h}^{\prime}\right)+\left(1-\beta_{h}\right) G_{l l}^{0}\left(\mathcal{C}_{l}^{\prime}\right) \\
> & \beta_{h}\left(\dot{\pi}_{h h} G_{h h}\left(\dot{\mathcal{C}}_{h}\right)+\dot{\pi}_{h l} G_{l h}^{0}\left(\dot{\mathcal{C}_{l}}\right)\right)+\beta_{l} G_{l l}^{0}\left(\dot{\mathcal{C}}_{l}\right) \\
= & \omega_{0}
\end{aligned}
$$

Therefore, we can deduce that allocation $\mathcal{Q}^{1}$ is dominated in $\mathbb{O}_{2}\left(\beta_{h}, c, \nu_{0}, \omega_{0}\right)$ because $\mathcal{Q}^{1}$ is feasible in $\mathbb{O}_{2}^{\prime}\left(\beta_{h}, c, \nu_{0}, \omega_{0}\right)$ with PUs' expected profit larger than $\omega_{0}$ and insureds' expected utility satisfying $R_{l l}\left(\mathcal{C}_{l}^{\prime}\right)=R_{l l}\left(\dot{\mathcal{C}}_{l}\right)$. Hence, we have a contradiction and in fact $\nu_{0}<\bar{\nu}_{0}$. Also, from (21) we can arrive at the conclusion that $R_{h h}\left(\dot{\mathcal{C}}_{h}\right)=R_{h l}\left(\dot{\mathcal{C}}_{l}, \dot{p}\right)=\nu_{0}$.

Remarks. Theorem 1 conveys that at the optimal solution to $\mathbb{O}_{2}\left(\beta_{h}, c, \nu_{0}, \omega_{0}\right)$ when $\dot{p}>0$, type- $h$ SUs randomize between contract $\dot{\mathcal{C}}_{l}$ and $\dot{\mathcal{C}}_{h}$ and selects $\dot{\mathcal{C}}_{l}$ with probability $B\left(\dot{\theta}_{l}, \beta_{h}, c\right)$. Moreover, the fact that $\nu_{0}<\bar{\nu}_{0}$ reveals that audition is an effective way to stem type- $h$ SUs from choosing contract $\dot{\mathcal{C}}_{l}$ because as indicated by Theorem 2, type- $h$ SUs' minimum utility $\nu_{0}$ is less than that granted by the pooling contract.

Proposition 1. For $\left\{\dot{\mathcal{C}}, \dot{p}, \dot{\pi}_{h}, \dot{\pi}_{l}\right\}$ such that $\dot{p}>0$, contract $\dot{\mathcal{C}}_{l}$ satisfies (30),

$$
\begin{aligned}
& \dot{\mathcal{C}}_{l}=\arg \max _{\mathcal{C}_{l}} R_{l l}\left(\mathcal{C}_{l}\right), \text { s.t. } \\
& \beta_{h}\left(B\left(\dot{\theta}_{l}, \beta_{h}, c\right)\left(G_{l h}^{0}\left(\dot{\mathcal{C}}_{l}\right)-G_{h h}\left(\dot{\mathcal{C}}_{h}\right)\right)+G_{h h}\left(\dot{\mathcal{C}}_{h}\right)\right)+ \\
& \left(1-\beta_{h}\right) G_{l l}^{0}\left(\dot{\mathcal{C}}_{l}\right) \geq \omega_{0}
\end{aligned}
$$

Proof: From Theorem 1, we get $\dot{\pi}_{l l}=1, \dot{\pi}_{l h}=0$ and (25). We get (30) by substituting $\dot{\pi}_{l l}, \dot{\pi}_{l h}$ and $\dot{\pi}_{h l}$ in (20).

Next, we further characterize the optimal solution of $\mathbb{O}_{3}\left(\beta_{h}, c, \bar{R}_{h h}, 0\right)=\left.\mathbb{O}_{2}\left(\beta_{h}, c, \nu_{0}, \omega_{0}\right)\right|_{\nu_{0}=\bar{R}_{h h}} ^{\omega_{0}=0}$, which is also a crucial step towards the characterization of $\mathbb{O}_{1} . \bar{R}_{h h}$ denotes a type- $h$ SU's utility if it signs an actuarially fair full insurance contract $\overline{\mathcal{C}}_{h}$ i.e., $\bar{R}_{h h}=R_{h h}\left(\overline{\mathcal{C}}_{h}\right)$. Then we have Theorem 3 characterizing the optimal solution to $\mathbb{O}_{3}$.
Theorem 3. There exist threshold values for PUs' audition cost $c^{*}\left(0<c^{*}<\infty\right)$ and the probability that an SUs falls into the category of type-h SU $\beta_{h}^{*}\left(0<\beta_{h}^{*}<1\right)$, such that the optimal solution for $\mathbb{O}_{3},\left\{\ddot{\mathcal{C}}, \ddot{p}, \ddot{\pi}_{h}, \ddot{\pi}_{l}\right\}$ satisfies,

- When $c^{*}<c<\infty$ and $\beta_{h}^{*}<\beta_{h}<1$, $\ddot{\mathcal{C}}=\left(\overline{\mathcal{C}}_{h}, \overline{\overline{\mathcal{C}}}_{l}\right), \ddot{\pi}_{h h}=\ddot{\pi}_{l l}=1$ and $\ddot{\pi}_{h l}=\ddot{\pi}_{l h}=0$.

- When $q\left(\beta_{h}\right) \leq c<\infty$ and $0<\beta_{h}<\beta_{h}^{*}$, $\ddot{\lambda}_{h}+\ddot{\theta}_{h}=L, \ddot{\lambda}_{l}+\ddot{\theta}_{l}<L, \ddot{\pi}_{h h}=\ddot{\pi}_{l l}=1$ and $\ddot{\pi}_{h l}=$ $\ddot{\pi}_{l h}=0$.

- When $0<c \leq c^{*}$ and $\beta_{h}^{*} \leq \beta_{h}<1$ or $0<c \leq q\left(\beta_{h}\right)$ and $0<\beta_{h}<\beta_{h}^{*}$,

$\ddot{\mathcal{C}}=\left(\overline{\mathcal{C}}_{h}, \dot{\mathcal{C}}_{l}^{\prime}\right), \ddot{\pi}_{h l}=B\left(\ddot{\theta}_{l}, \beta_{h}, c\right), \ddot{\pi}_{h h}=1-\ddot{\pi}_{h l}, \ddot{\pi}_{l l}=1$ and $\ddot{\pi}_{l h}=0$.

where $\overline{\overline{\mathcal{C}}_{l}}=\left\{\xi_{l} L^{\prime},\left(1-\xi_{l}\right) L^{\prime}\right\}\left(0<L^{\prime}<L\right)$, curve $c=$ $q\left(\beta_{h}\right)\left(0<\beta_{h} \leq \beta_{h}^{*}\right)$ is shown in Figure 2 and $\dot{\mathcal{C}}_{l}^{\prime}$ satisfies:

$$
\begin{aligned}
& \dot{\mathcal{C}}_{l}^{\prime}=\arg \max _{\mathcal{C}_{l}} R_{l l}\left(\mathcal{C}_{l}\right), \text { s.t. } \\
& \beta_{h} B\left(\dot{\theta}_{l}^{\prime}, \beta_{h}, c\right) G_{l h}^{0}\left(\dot{\mathcal{C}}_{l}^{\prime}\right)+\left(1-\beta_{h}\right) G_{l l}^{0}\left(\dot{\mathcal{C}}_{l}^{\prime}\right) \geq 0 .
\end{aligned}
$$

Proof: By substituting $\overline{\mathcal{C}}_{h}=\left\{\xi_{h} L, L-\xi_{h} L\right\}$ into (8), we have $G_{h h}\left(\overline{\mathcal{C}}_{h}\right)=0$. Together with $\omega_{0}=0$, we get condition (31). Then, we denote the value function of $\mathbb{O}_{3}\left(\beta_{h}, c, \bar{R}_{h h}, 0\right)$ as $\psi_{3}\left(\beta_{h}, c, \bar{R}_{h h}, 0\right)=\left.\psi_{2}\left(\beta_{h}, c, \nu_{0}, \omega_{0}\right)\right|_{\nu_{0}=\bar{R}_{h h}} ^{\omega_{0}=0}$.

If $\ddot{p}=0, R_{l l}\left(\ddot{C}_{l}\right)$ is contingent merely on the value of $\beta_{h}$ and thus, in this scenario, we can denote that $k\left(\beta_{h}\right)=R_{l l}\left(\ddot{\mathcal{C}}_{l}\right)$. According to the Rothschild-Stiglitz (RS) model [19], a threshold value $\beta_{h}^{*}\left(0<\beta_{h}^{*}<1\right)$ exists satisfying that $k\left(\beta_{h}\right)>R_{l l}\left(\overline{\bar{C}}_{l}\right)$ and $k^{\prime}\left(\beta_{h}\right)<0$ for $0 \leq \beta_{h}<\beta_{h}^{*}$ and $k\left(\beta_{h}\right)=R_{l l}\left(\overline{\bar{C}}_{l}\right)$ for $\beta_{h}^{*} \leq \beta_{h}<1$. Since $\dot{\mathcal{C}}_{l}^{\prime}$ depends only on cost $c$, we can denote that $t(c)=R_{l l}\left(\dot{\mathcal{C}}_{l}^{\prime}\right)$ such that $t^{\prime}(c)<0$. According to the RS model in [19], a threshold value $c^{*}\left(0<c^{*}<\infty\right)$ exists such that $t(c)>R_{l l}\left(\overline{\bar{C}}_{l}\right)$ if and only if when $0<c<c^{*}$. Based on the aforementioned properties of $k\left(\beta_{h}\right)$ and $t(c)$, we have:

- When $c^{*}<c<\infty$ and $\beta_{h}^{*}<\beta_{h}<1$, $\psi_{3}\left(\beta_{h}, c, \bar{R}_{h h}, 0\right)=k\left(\beta_{h}\right)=R_{l l}\left(\overline{\bar{C}}_{l}\right)>R_{l l}\left(\dot{\mathcal{C}}_{l}^{\prime}\right)$.

- When $q\left(\beta_{h}\right) \leq c<\infty$ and $0<\beta_{h}<\beta_{h}^{*}$, $\psi_{3}\left(\beta_{h}, c, \bar{R}_{h h}, 0\right)=k\left(\beta_{h}\right)>\max \left\{R_{l l}\left(\dot{\mathcal{C}}_{l}^{\prime}\right), R_{l l}\left(\overline{\bar{C}}_{l}\right)\right\}$.

- When $0<c \leq c^{*}$ and $\beta_{h}^{*} \leq \beta_{h}<1$ or $0<c \leq q\left(\beta_{h}\right)$ and $0<\beta_{h}<\beta_{h}^{*}$,

$$
\psi_{3}\left(\beta_{h}, c, \bar{R}_{h h}, 0\right)=t(c)=R_{l l}\left(\dot{\mathcal{C}}_{l}^{\prime}\right)>k\left(\beta_{h}\right) .
$$

Then, together with Theorems 1 and 2, we get Theorem 3 concerning the optimal solution of $\mathbb{O}_{3}$.

Remarks. In Theorem 3, we characterize the contract forms and SUs' strategies at the solution to $\mathbb{O}_{3}\left(\beta_{h}, c, \bar{R}_{h h}, 0\right)$. It reveals that threshold values $c^{*}\left(0<c^{*}<\infty\right)$ and $\beta_{h}^{*}(0<$ $\left.\beta_{h}^{*}<1\right)$, together with the function $c=q\left(\beta_{h}\right)$ divide the area $\mathcal{S}=\left\{\left(\beta_{h}, c\right) \mid 0<\beta_{h}<1\right.$ and $\left.0<c<\infty\right\}$ in the $c-\beta_{h}$ plane into three regions shown in Figure 2. In region I and II, the contract forms are specified in the first two items of Theorem 3 and SUs strongly prefer the contacts designed for their own risk types. Hence, audition is not necessary, i.e., $\ddot{p}=0$. When $\left(\beta_{h}, c\right)$ falls into region III, then for PUs the optimal contracts are in the form of $\ddot{\mathcal{C}}=\left(\overline{\mathcal{C}}_{h}, \dot{\mathcal{C}}_{l}^{\prime}\right)$ and audition is conducted with probability $\ddot{p}>0$. In this case, type-l SUs also strongly 


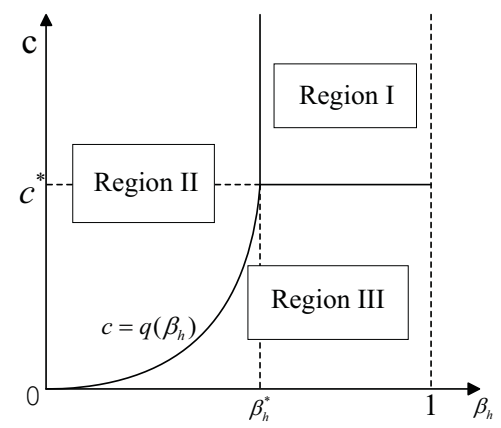

Fig. 2. Illustration of $c-\beta_{h}$ plane: $c^{*}$ and $\beta_{h}^{*}$ are threshold values.

prefer the contacts designed for type- $l$ SUs, whereas type- $h$ SUs select $\dot{\mathcal{C}}_{l}^{\prime}$ with probability $\ddot{\pi}_{h l}=B\left(\ddot{\theta}_{l}, \beta_{h}, c\right)$.

After we propose Theorems 1-3, we can characterize the optimal solution to $\mathbb{O}_{1}\left(\beta_{h}, c, \nu_{0}, \omega_{1}, \cdots, \omega_{m}\right)$ defined in (24). Specifically, we consider the scenario where PUs obtain nonnegative profit that is we consider $\mathbb{O}_{1}\left(\beta_{h}, c, \nu_{0}, 0, \cdots, 0\right)$.

Theorem 4. The optimal solution to $\mathbb{O}_{1}\left(\beta_{h}, c, \nu_{0}, 0, \cdots, 0\right)$ coincides with the optimal solution to $\mathbb{O}_{2}\left(\beta_{h}, c, \nu_{0}, 0\right)$ characterized in Theorems 1-3, that is for $\forall i \in\{1, \cdots, m\}$, we have $\widehat{\mathcal{C}}_{i}=\dot{\mathcal{C}}_{i}, \widehat{p}_{i}=\dot{p}_{i}, \widehat{\pi}_{j k}^{i}=\frac{\dot{\pi}_{j k}}{m}, j, k \in\{h, l\}$ under the condition that $\nu_{0}^{\prime} \neq \bar{R}_{h h}$ where $\nu_{0}^{\prime}$ denotes type- $h$ insureds' expected utility i.e., $\nu_{0}^{\prime}=\Lambda_{h}\left(\mathbf{C}, \mathbf{p}, \pi_{h}\right)$.

Proof: The proof of Theorem 4 is in the appendix.

\section{Characterization of Perfect Bayesian}

\section{EQUILIBRIUM OF THE FOUR-STAGE BAYESIAN GAME}

In this section, we seek to characterize the equilibrium of the four-stage Bayesian game and restrict our analysis to equilibrium allocations that are SBPO. A contradiction with the definition of equilibrium will occur if a non-SBPO allocation is an equilibrium allocation because any arbitrary deviant PU $k$ will then be able to offer a contract menu $\mathcal{C}_{k}=\left(\mathcal{C}_{h}^{k}, \mathcal{C}_{l}^{k}\right)$ satisfying that this contract menu will be better off to all SUs and meanwhile PU $k$ makes positive profit.

Then, we denote $\Lambda_{h}^{e}$ and $\Lambda_{l}^{e}$ respectively as the equilibrium expected utility for type- $h$ and type- $l$ SUs. Then, in $\mathbb{C}_{2}$ and $\mathbb{C}_{3}$, we introduce two additional constraints incorporated in the following analysis.

$\mathbb{C}_{2}$ : A PBE $\mathcal{E}=\left\{\mathbf{C}^{*}, \mathbf{p}^{*}(\cdot), \pi_{h}^{*}(\cdot), \pi_{l}^{*}(\cdot), \eta^{*}(\cdot)\right\}$ satisfies $\mathbb{C}_{2}$ if for the continuation sub-game after PUs propose the contract profile $\mathbf{C}^{*},\left\{\mathbf{p}^{*}, \pi_{h}^{*}, \pi_{l}^{*}\right\}$ is a trembling hand perfect Bayesian equilibrium introduced by Selten in [20].

$\mathbb{C}_{3}:$ A PBE $\mathcal{E}=\left\{\mathbf{C}^{*}, \mathbf{p}^{*}(\cdot), \pi_{h}^{*}(\cdot), \pi_{l}^{*}(\cdot), \eta_{i}^{*}(\cdot)\right\}$ satisfies $\mathbb{C}_{3}$ if $\pi_{l}^{*}(\mathbf{C})=\pi_{l}^{*}\left(\mathbf{C}^{\prime}\right)$ such that contract profile $\mathbf{C}$ and $\mathbf{C}^{\prime}$ differs only in the contracts strictly dominated for type-l SUs.

Then we have Lemma 3 to characterize basic properties of PBE that satisfies constraint $\mathbb{C}_{2}$.

Lemma 3. We have $p_{i}^{*}=0$ for $\forall i \in\{1, \cdots, m\}$ such that $\theta_{l}^{i *}=c$ at any $\operatorname{PBE} \mathcal{E}=\left\{\mathbf{C}^{*}, \mathbf{p}^{*}(\cdot), \pi_{h}^{*}(\cdot), \pi_{l}^{*}(\cdot), \eta_{i}^{*}(\cdot)\right\}$ that satisfies $\mathbb{C}_{2}$.

Proof: We denote $\Pi$ as the continuation subgame after the proposal of $\mathbf{C}^{*}$ and $\left\{\mathbf{p}^{*}, \pi_{h}^{*}, \pi_{l}^{*}\right\}$ is a perfect Bayesian equilibrium of $\Pi$ that satisfies $\mathbb{C}_{2}$. We consider a sequence of perturbed continuation subgames $\left.\left\{\Pi_{\varepsilon_{n}}\right\}\right|_{n=1} ^{\infty}$ which converges to $\Pi$ and the associated PBE $\left\{\mathbf{p}_{n}, \pi_{h n}, \pi_{l n}, \eta_{i n}\right\}$ satisfying that $\left\{\mathbf{p}_{n}, \pi_{h n}, \pi_{l n}\right\}$ converges to $\left\{\mathbf{p}^{*}, \pi_{h}^{*}, \pi_{l}^{*}\right\}$. Then, from Bayes law, we have: $\eta_{n}^{i}=\frac{\beta_{h} \xi_{h} \pi_{h l n}^{i}}{\beta_{h} \xi_{h} \pi_{h l n}^{i}+\left(1-\beta_{h}\right) \xi_{l} \pi_{l l n}^{i}} \in(0,1), \forall n$. Maximizing $p_{i}\left(\eta_{n}^{i} \theta_{l}^{i *}-c\right)$ w.r.t. $p_{i} \in\left[\varepsilon_{n}, 1-\varepsilon_{n}\right]$ means that if $\theta_{l}^{i *}=c, \forall n$, we have $p_{i n}=\varepsilon_{n}$. Therefore, the fact that $\lim _{n \rightarrow \infty} \varepsilon_{n}=0$ reveals that $p_{i}=\lim _{n \rightarrow \infty} p_{i n}=0$.

Remarks. Lemma 3 reveals that under constraint $\mathbb{C}_{2}$, the decrease in the audition probability of PU $i$ is not able to serve as a threat to deter an deviant PU to attract type- $h$ SUs. Thus any PBE allocation falls into the category of SBPO allocation, which is formally proved in Lemma 4.

Lemma 4. Any PBE allocation satisfying $\mathbb{C}_{2}$ is SBPO and an optimal solution to $\mathbb{O}_{1}\left(\beta_{h}, c, \Lambda_{h}^{e}, 0, \cdots, 0\right)$ with $\Lambda_{h}^{e} \leq \bar{R}_{h h}$.

Proof: Assume that $\mathcal{E}=\left\{\mathbf{C}^{*}, \mathbf{p}^{*}(\cdot), \pi_{h}^{*}(\cdot), \pi_{l}^{*}(\cdot), \eta_{i}^{*}(\cdot)\right\}$ is a PBE and its allocation $\left\{\mathbf{C}^{*}, \mathbf{p}^{*}, \pi_{h}^{*}, \pi_{l}^{*}\right\}$ satisfies $\mathbb{C}_{2}$. $\left\{\mathbf{C}^{*}, \mathbf{p}^{*}, \pi_{h}^{*}, \pi_{l}^{*}\right\}$ is feasible in $\mathbb{O}_{1}\left(\beta_{h}, c, \Lambda_{h}^{e}, \Omega_{j}^{e}, \cdots, \Omega_{j}^{e}\right)$ with $\Omega_{i}^{e}$ denoting the equilibrium expected revenue of $\mathrm{PU} i$, for $\forall i \in\{1, \cdots, m\}$. Since $\Omega_{i}^{e} \geq 0$, we have $\left\{\mathbf{C}^{*}, \mathbf{p}^{*}, \pi_{h}^{*}, \pi_{l}^{*}\right\}$ is also feasible in $\mathbb{O}_{1}\left(\beta_{h}, c, \Lambda_{h}^{e}, 0, \cdots, 0\right)$.

Then we also prove this lemma by contradiction. We assume that this allocation does not optimize $\mathbb{O}_{1}\left(\beta_{h}, c, \Lambda_{h}^{e}, 0, \cdots, 0\right)$. We consider a deviation made by $\mathrm{PU} j$ from $\mathcal{C}_{j}^{*}$ to $\mathcal{C}_{j}$. We denote $\Omega_{j}$ as PU $j$ 's profit of the continuation equilibrium after its deviation. Hence, $\Omega_{j}$ and $\Omega_{j}^{e}$ satisfies (15) that is $\Omega_{j}<\Omega_{j}^{e}$. In the scenario where $R_{h l}^{\prime}\left(\mathcal{C}_{l}^{j}\right)<R_{h h}\left(\mathcal{C}_{h}^{j}\right)$, we have $\pi_{h h}^{*}\left(\mathcal{C}_{j}, \mathbf{C}_{-j}^{*}\right)=\pi_{l l}^{*}\left(\mathcal{C}_{j}, \mathbf{C}_{-j}^{*}\right)=1$ and $p_{j}^{*}\left(\mathcal{C}_{j}, \mathbf{C}_{-j}^{*}\right)=0$. Hence, $\Omega_{j} \geq \Omega_{j}^{e}$ which contradicts with $\Omega_{j}<\Omega_{j}^{e}$. Also similar results hold in the case that $R_{h l}^{\prime}\left(\mathcal{C}_{l}^{j}\right) \geq R_{h h}\left(\mathcal{C}_{h}^{j}\right)$.

After Lemmas 3 and 4 , we are ready to characterize the PBE of this four-stage Bayesian game satisfying $\mathbb{C}_{2}$ and $\mathbb{C}_{3}$. In Theorem 5 , we propose the necessary and sufficient conditions for PBE to exist in this four-stage Bayesian game.

Theorem 5. Under $\mathbb{C}_{2}$ and $\mathbb{C}_{3}$ only two types of PBE exist:

- If and only if $0<c \leq c^{*}$ and $\beta_{h}^{*} \leq \beta_{h}<1$ or $0<c \leq$ $k\left(\beta_{h}\right)$ and $0<\beta_{h}<\beta_{h}^{*}$,

PBE $\mathcal{E}_{1}$ exists at which PUs propose contract menu $\mathcal{C}_{i}^{*}=\left(\dot{\mathcal{C}}_{l}^{\prime}, \overline{\mathcal{C}}_{h}\right), \forall i \in\{1, \cdots, m\}$ and carry out audition with positive probability. Type-l SUs strongly prefer $\dot{\mathcal{C}}_{l}^{\prime}$ whereas type- $h$ SUs randomize between $\dot{\mathcal{C}}_{l}^{\prime}$ and $\overline{\mathcal{C}}_{h}$.

- If and only if $\beta_{h}^{*}<\beta_{h}<1$ and $c^{*}<c<\infty$, $\mathrm{PBE} \mathcal{E}_{2}$ exists where PUs propose contract menu $\mathcal{C}_{i}^{*}=$ $\left(\overline{\overline{\mathcal{C}}}_{l}, \overline{\mathcal{C}}_{h}\right), \forall i \in\{1, \cdots, m\}$ and SUs only select contracts designed for their own risk types. Thus, audition is not necessary, i.e., $p_{i}^{*}=0$.

Proof: The proof of Theorem 5 is in the appendix.

\section{Numerical and Simulation Results}

In this section, we provide our numerical and simulation results. We demonstrate the existence of $\operatorname{PBE} \mathcal{E}_{1}$ and $\mathcal{E}_{2}$ in Figures 3 and 4. Moreover, in Figure 5, we demonstrate the comparison of the utility of an SU with and without insurance. Without loss of generality we set the simulation parameters as follows: $U_{S}=60, U_{F}=2, \tau=1, \xi_{h}=0.7$ and $\xi_{l}=0.3$. 


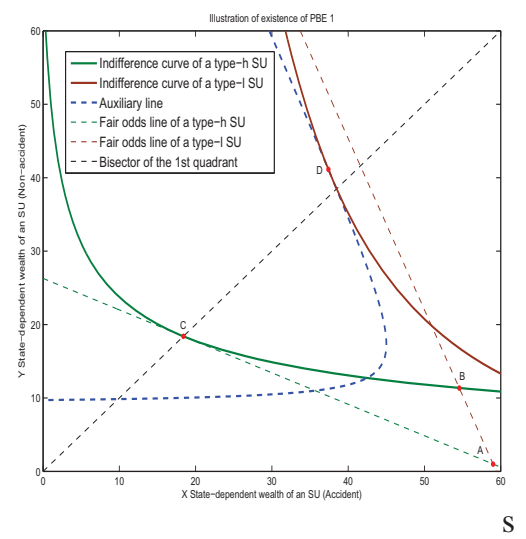

Fig. 3. Illustration of existence of PBE 1 .

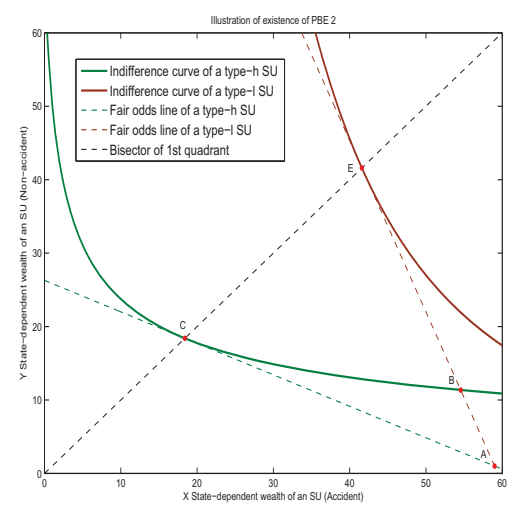

Fig. 4. Illustration of existence of PBE 2.

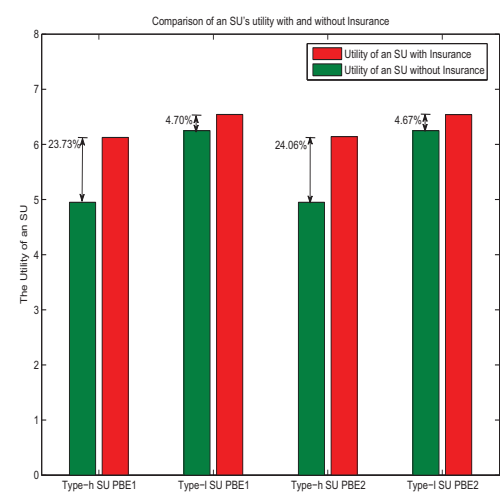

Fig. 5. Comparison of an SU's utility with and without insurance.
In Figure 3, the horizontal axis represents the statedependent wealth of an SU when an accident does not occur to it and the vertical axis is the state-dependent wealth of an SU when an accident happens to this particular SU. Then point $A(59,1)$ represents the combination of the state-dependent wealth of an SU when it does not purchase any insurance contract. Moreover, the dashed line $A C$ and $A B$ respectively represents the fair odds line of a type- $h$ SU and a type-l SU. For a type- $j \mathrm{SU}$, its fair odds line goes through point $A(59,1)$ with slope $\frac{\xi_{j}-1}{\xi_{j}}, j \in\{l, h\}$. From Figure 3, we notice that for type- $h$ SU one of its indifference curves is tangent to its fair-odds line at point $C(18.4,18.4)$ which is the intersection of line $A C$ and the bisector of 1st quadrant. Thus, we can conclude that at PBE $\mathcal{E}_{1}$ the contract designed for type- $h \mathrm{SU}$ is $\overline{\mathcal{C}}_{h}=\{40.6,17.4\}=\left\{\xi_{h} L, L-\xi_{h} L\right\}$. Furthermore, from (28) and (30), we can calculate that contract $\mathcal{C}_{l}=\left(\lambda_{l}, \theta_{l}\right)$ breaks even when $\lambda_{l}=\frac{0.21 \theta_{l}^{2}}{0.49 \theta_{l}-0.4 c}$. Without loss of generality, we set $c=10$ in our simulation and we additionally draw an ancillary curve $x=59-\frac{0.21(y-1)^{2}}{0.49(y-1)-4}$ shown in Figure 3 as the dashed blue curve. Also, as shown in Figure 3, we can find an indifference curve for type-l SU tangent to the ancillary curve at point $D(37.41,41.13)$. Hence, at $\operatorname{PBE} \mathcal{E}_{1}$, the insurance contract designed for type- $l$ SUs is $\dot{\mathcal{C}}_{l}^{\prime}=(21.59,40.13)$. We have shown the existence of PBE $\mathcal{E}_{1}$ in Figure 3.

Similarly, in Figure 4, we demonstrate the existence of PBE $\mathcal{E}_{2}$. For $\operatorname{PBE} \mathcal{E}_{2}$, we do not need to find the point where one of the type-l SUs' indifference curves is tangent to the ancillary curve in Figure 3. Since line $A E$, the fair odds line of a type- $l$ $\mathrm{SU}$ intersects with the indifference curve of type- $h \mathrm{SU}$ at point $B(54.56,11.36)$, we can conclude that that $\mathrm{PBE} \mathcal{E}_{2}$ exists and the PBE contracts designed for type- $l$ SUs and type- $h$ SUs are respectively $\overline{\overline{\mathcal{C}}}=\{4.44,10.36\}$ and $\overline{\mathcal{C}}_{h}=\{40.6,17.4\}$.

Furthermore, based on the aforementioned results about the contractual forms and SUs' contract choices at PBE $\mathcal{E}_{1}$ and $\operatorname{PBE} \mathcal{E}_{2}$. We can calculate the expected utility of an SU in at both PBEs and compare them with the expected utility of an $\mathrm{SU}$ when insurance is not introduced into spectrum trading in CR network. Thus, in Figure 5, we show the comparison of an
SU's expected utility with and without insurance. Apparently, from Figure 5, we can conclude that the SUs experience significant increase in their utilities if they choose to purchase insurance contracts at both PBEs.

\section{Vi. Conclusions and Future Work}

In this paper, we utilize insurance theory in spectrum trading in CRNs and model the market game as a four-stage Bayesian game. We show that at PBE, both PUs and SUs will experience increase in their utilities. This indicates by signing insurance contracts, SUs will have more incentives to buy the channels and PUs can make non-negative profit from every contract it offers. Thus, spectrum utilization in CRNs can be improved. In future work, we aim to analyze the scenario where more than two types of SUs exist and analyze the related equilibrium. We aim also to study the case where channels are heterogeneously priced by PUs and study the effects of different channel pricing strategies on the forms of the insurance contracts.

\section{REFERENCES}

[1] A. Gopinathan, Z. Li, "Strategyproof auctions for balancing social welfare and fairness in secondary spectrum markets," in Proceedings of IEEE INFOCOM, 2011, pp.3020-3028, 10-15 April 2011

[2] L. Deek, X. Zhou, K. Almeroth, and H. Zheng, "To preempt or not: Tackling bid and time-based cheating in online spectrum auctions," in Proceedings of IEEE INFOCOM 2011, pp. 2219-2227, april 2011.

[3] H. Kim, J. Choi, K.G Shin, "Wi-Fi 2.0: Price and quality competitions of duopoly cognitive radio wireless service providers with timevarying spectrum availability," in Proceedings of IEEE INFOCOM, 2011, pp.2453-2461, 10-15 April 2011

[4] A. Gopinathan, Z. Li, "A prior-free revenue maximizing auction for secondary spectrum access," IEEE INFOCOM,, pp.86-90, April 2011

[5] L. Duan, J. Huang, B. Shou, "Cognitive Mobile Virtual Network Operator: Investment and Pricing with Supply Uncertainty," in Proceedings of IEEE INFOCOM, 2010,, pp. 1-9, March 2010

[6] X. Zhou and H. Zheng, "Trust: A general framework for truthful double spectrum auctions," IEEE INFOCOM 2009, pp. 999-1007, april 2009.

[7] L. Duan, L. Gao, and J. Huang, "Contract-based cooperative spectrum sharing," in IEEE DySPAN, 2011, pp. 399-407, 2011.

[8] L. Gao, X. Wang, Y. Xu, Q. Zhang, "Spectrum Trading in Cognitive Radio Networks: A Contract-Theoretic Modeling Approach," IEEE Journal on Selected Areas in Communications, , pp.843-855, April 2011

[9] D. Niyato, E. Hossain, "Competitive Pricing for Spectrum Sharing in Cognitive Radio Networks: Dynamic Game, Inefficiency of Nash Equilibrium, and Collusion," IEEE Journal on Selected Areas in Communications,vol.26, no.1, pp.192-202, Jan. 2008 
[10] L. Gao, Y. Xu, X. Wang, "MAP: Multiauctioneer Progressive Auction for Dynamic Spectrum Access," IEEE Transactions on Mobile Computing, vol. 10, no. 8, pp. 1144-1161, Aug. 2011

[11] L. Duan, J. Huang, B. Shou, "Investment and Pricing with Spectrum Uncertainty: A Cognitive Operators Perspective," IEEE Transactions on Mobile Computing, vol. 10, no. 11, pp. 1590-1604, Nov. 2011

[12] D. Niyato, E. Hossain, Z. Han, "Dynamics of Multiple-Seller and Multiple-Buyer Spectrum Trading in Cognitive Radio Networks: A Game-Theoretic Modeling Approach," IEEE Transactions on Mobile Computing. vol.8, no.8, pp.1009-1022, Aug. 2009

[13] M. Garetto and E. Leonardi, "Restricted mobility improves delaythroughput tradeoffs in mobile ad hoc networks," IEEE Transactions on Information Theory, vol. 56, no. 10, pp. 5016 -5029, oct. 2010.

[14] X. Zhou, S. Gandhi, S. Suri, and H. Zheng, "Ebay in the sky: strategyproof wireless spectrum auctions," in Proceedings of the 14th ACM international conference on Mobile computing and networking, 2008.

[15] J. Jia, Q. Zhang, Q. Zhang, and M. Liu, "Revenue generation for truthful spectrum auction in dynamic spectrum access," in Proceedings of the tenth ACM international symposium on Mobile ad hoc networking and computing. pp. 3-12, 2009.

[16] M. Clarke, and G. Légiste, "The law of insurance contracts". Lloyd's of London Press, 1989.

[17] J. Schiller, "The impact of insurance fraud detection systems," Journal of Risk and Insurance, vol. 73, no. 3, pp. 421-438, 2006.

[18] P. Picard, "Costly risk verification without commitment in competitive insurance markets," Games and Economic Behavior, pp. 893-919, 2009.

[19] M. Rothschild and J. Stiglitz, "Equilibrium in competitive insurance markets: An essay on the economics of imperfect information," The Quarterly Journal of Economics, vol. 90, no. 4, pp. 629-649, 1976.

[20] R. Selten, "Reexamination of the perfectness concept for equilibrium points in extensive games," International journal of game theory, 1975.

\section{ACKNOWLEDGMENT}

This work is supported by National Fundamental research grant (2011CB302701), NSF China (No. 60832005); China Ministry of Education New Century Excellent Talent (No. NCET-10-0580); China Ministry of Education Fok Ying Tung Fund (No. 122002); Qualcomm Research Grant; Shanghai Basic Research Key Project (No.11JC1405100); National Key Project of China (2010ZX03003-001-01).

\section{APPENDIX}

\section{A. Proof of Theorem 4}

Proof: We denote $\psi_{1}\left(\beta_{h}, c, \nu_{0}, 0\right)$ as the value function of $\mathbb{O}_{1}\left(\beta_{h}, c, \nu_{0}, 0, \cdots, 0\right)$. Also, we denote $\widetilde{\psi}_{2}\left(\beta_{h}, c, \nu_{0}, 0\right)$ as the value function of $\mathbb{O}_{2}\left(\beta_{h}, c, \nu_{0}, \omega_{0}\right)$ with $\omega_{0}=0$ and (20) is changed from inequality to equality. We denote $\beta_{h}^{i}$ as the fraction of type- $h$ SUs among SUs that buy PU $i$ 's insurance.

- When $\nu_{0}^{\prime}>\bar{R}_{h h}$

From (20) and condition $\Lambda_{h}\left(\widehat{\mathbf{C}}, \widehat{\mathbf{p}}, \widehat{\pi}_{h}\right) \geq \nu_{0}$ in (24), we have $\beta_{i}<1$. From the definitions of $\psi_{2}$ and $\widetilde{\psi}_{2}$, we get

$$
\widetilde{\psi}_{2}\left(\beta_{h}^{i}, c, \nu_{0}^{\prime}, 0\right) \geq \psi_{1}\left(\beta_{h}, c, \nu_{0}, 0\right) \geq \widetilde{\psi}_{2}\left(\beta_{h}^{i}, c, \nu_{0}, 0\right)
$$

Since $\widetilde{\psi}_{2}$ decreases if and only if $\nu_{0}<R_{h h}^{0}=R_{h h}(0,0)$ and $\nu_{0}^{\prime}>\bar{R}_{h h}$, we have $\beta_{h}^{i} \leq \beta_{h}$. We denote that $N_{i}=$ $\widehat{\pi}_{h h}^{i}+\widehat{\pi}_{h l}^{i}+\widehat{\pi}_{l h}^{i}+\widehat{\pi}_{l l}^{i}$ which is the proportion of SUs buying contracts from PU $i$. Hence, we have $\beta_{h}=\sum_{i=0}^{m} \beta_{h}^{i} N_{i}$ and $\sum_{i=0}^{m} N_{i}=1$. We get the conclusion that $\beta_{h}^{i}=\beta_{h}$.

- When $\nu_{0}^{\prime}<\bar{R}_{h h}$

We can get similar results using similar analysis as before.

Thus, in the case where $\nu_{0}^{\prime} \neq \bar{R}_{h h}$, we have $\beta_{h}^{i}=\beta_{h}$ and

$\psi_{1}\left(\beta_{h}, c, \nu_{0}, 0\right)=\psi_{2}\left(\beta_{h}, c, \nu_{0}, 0\right)=\widehat{\pi}_{l h}^{i} R_{l l}\left(\widehat{\mathcal{C}}_{h}^{i}\right)+\widehat{\pi}_{l l}^{i} R_{l l}\left(\widehat{\mathcal{C}}_{l}^{i}\right)$
Therefore, for $\forall i \in\{1, \cdots, m\}$ such that $N_{i}>0$, $\left\{\widehat{\mathcal{C}}_{i}, \widehat{p}_{i}, \widehat{\pi}_{h h}^{i}, \widehat{\pi}_{h l}^{i}, \widehat{\pi}_{l l}^{i}, \widehat{\pi}_{l h}^{i}\right\}$ is feasible in $\mathbb{O}_{2}\left(\beta_{h}, c, \nu_{0}, 0\right)$ and it reveals that the optimal solution to $\mathbb{O}_{1}\left(\beta_{h}, c, \nu_{0}, 0, \cdots, 0\right)$ does coincide with the one to $\mathbb{O}_{2}\left(\beta_{h}, c, \nu_{0}, 0\right)$ when $\nu_{0}^{\prime} \neq \bar{R}_{h h}$.

\section{B. Proof of Theorem 5}

Proof: Suppose $\widehat{\nu}_{0}=\left\{\nu_{0} \mid \psi_{2}\left(\beta_{h}, c, \nu_{0}, \omega_{0}\right) \geq R_{l l}\left(\mathcal{C}_{l}^{0}\right)\right\}$. If $R_{h h}^{0} \leq \nu_{0}<\widehat{\nu}_{0}$, we have $\psi_{1}\left(\beta_{h}, c, \nu_{0}, 0\right)=\psi_{2}\left(\beta_{h}, c, \nu_{0}, 0\right)$. Hence, $\psi_{1}\left(\beta_{h}, c, \nu_{0}, 0\right)>R_{l l}\left(\mathcal{C}_{l}^{0}\right)$. Then, we conclude that if $R_{h h}^{0} \leq \nu_{0}<\widehat{\nu}_{0}$, each SU signs an insurance contract at the optimal solution to $\mathbb{O}_{1}\left(\beta_{h}, c, \nu_{0}, 0\right)$. From Lemma 4 , we have $\Lambda_{h}^{e} \leq \bar{R}_{h h}$. Under $\mathbb{C}_{3}$, we have $\Lambda_{h}^{e}=\bar{R}_{h h}$. We get at PBE that satisfies $\mathbb{C}_{2}$ and $\mathbb{C}_{3}$, each SU purchases an insurance contract.

We prove the first case of Theorem 5. Since we consider PBE under $\mathbb{C}_{2}$, Lemmas 3 and 4 reveal $\psi_{3}\left(\beta_{h}, c, \bar{R}_{h h}, 0\right)=$ $R_{l l}\left(\dot{\mathcal{C}}_{l}^{\prime}\right)$ is a necessary condition for PBE $\mathcal{E}_{1}$ to exist. We seek to prove it is also a sufficient condition. Hence, we assume $\psi_{3}\left(\beta_{h}, c, \bar{R}_{h h}, 0\right)=R_{l l}\left(\dot{\mathcal{C}}_{l}^{\prime}\right)$ and $\left\{\mathbf{C}^{*}, \mathbf{p}^{*}, \pi_{h}^{*}, \pi_{l}^{*}\right\}$ is the PBE allocation characterized in this case. We consider the case where PU $i$ deviates from $\mathbf{C}^{*}$ by offering $\mathcal{C}_{i}=\left(\mathcal{C}_{h}^{i}, \mathcal{C}_{l}^{i}\right)$ instead of $\mathcal{C}_{i}^{*}=\left(\mathcal{C}_{h}^{i *}, \mathcal{C}_{l}^{i *}\right)$. In the continuation subgame after the proposal of $\left(\mathcal{C}_{i}, \mathbf{C}_{-i}^{*}\right) \neq \mathbf{C}^{*}, \mathbf{p}=\mathbf{p}^{*}\left(\mathcal{C}_{i}, \mathbf{C}_{-i}^{*}\right), \pi_{h}=\pi_{h}^{*}\left(\mathcal{C}_{i}, \mathbf{C}_{-i}^{*}\right)$, $\pi_{l}=\pi_{l}^{*}\left(\mathcal{C}_{i}, \mathbf{C}_{-i}^{*}\right)$ and $\eta_{i}=\eta_{i}^{*}\left(\mathcal{C}_{i}, \mathbf{C}_{-i}^{*}\right)$ can be chosen such that (13)-(15) and (17) hold. Then we have to prove that (16) also holds. We denote the expected profit of PU $i$ obtained from an insurance contract after the proposal of $\left(\mathcal{C}_{i}, \mathbf{C}_{-i}^{*}\right)$ as $\Omega_{i}^{\prime}=\Omega_{i}^{\prime}\left(\left(\mathcal{C}_{i}, \mathbf{C}_{-i}^{*}\right), \mathbf{p}^{*}\left(\mathcal{C}_{i}, \mathbf{C}_{-i}^{*}\right), \pi_{h}^{*}\left(\mathcal{C}_{i}, \mathbf{C}_{-i}^{*}\right), \pi_{l}^{*}\left(\mathcal{C}_{i}, \mathbf{C}_{-i}^{*}\right)\right)$ and we assume $\Omega_{i}^{e}=0$, so (17) can be written as $\Omega_{i}^{\prime} \leq 0$.

- When $\max \left\{R_{l l}\left(\mathcal{C}_{l}^{i}\right), R_{l h}\left(\mathcal{C}_{h}^{i}\right)\right\} \leq R_{l l}\left(\mathcal{C}_{l}^{\prime}\right)$

We can construct the continuation equilibrium such that $\pi_{l h}^{i}+\pi_{l l}^{i}=0$ which reveals that $\Omega_{i}^{\prime} \leq 0$.

- When $\max \left\{R_{l l}\left(\mathcal{C}_{l}^{i}\right), R_{l h}\left(\mathcal{C}_{h}^{i}\right)\right\}>R_{l l}\left(\dot{\mathcal{C}}_{l}^{\prime}\right)$ We can choose $\pi_{l h}^{i}$ and $\pi_{l l}^{i}$ such that $\pi_{l h}^{i}+\pi_{l l}^{i}=1$ and $\pi_{l h}^{i} R_{l h}\left(\mathcal{C}_{h}^{i}\right)+\pi_{l l}^{i} R_{l l}\left(\mathcal{C}_{l}^{i}\right)=\max \left\{R_{l l}\left(\mathcal{C}_{l}^{i}\right), R_{l h}\left(\mathcal{C}_{h}^{i}\right)\right\}$.

If $\pi_{l h}^{i} R_{l h}\left(\mathcal{C}_{h}^{i}\right)+\pi_{l l}^{i} R_{l l}\left(\mathcal{C}_{l}^{i}\right)<0$, we have $\Omega_{i}^{\prime}<0$. Otherwise, we consider two cases: $R_{h h}\left(\mathcal{C}_{h}^{i}\right) \geq \bar{R}_{h h}$ and $R_{h h}\left(\mathcal{C}_{h}^{i}\right)<\bar{R}_{h h}$.

- When $R_{h h}\left(\mathcal{C}_{h}^{i}\right) \geq \bar{R}_{h h}$ We construct the continuation equilibrium after $\left(\mathcal{C}_{i}, \mathbf{C}_{-i}^{*}\right)$ is proposed. Additionally, if $R_{h l}^{\prime}\left(\mathcal{C}_{l}^{i}\right)>R_{h h}\left(\mathcal{C}_{h}^{i}\right), \theta_{l}^{i}>c$ and $B\left(\theta_{l}^{i}, \beta_{h}, c\right) \pi_{l l}^{i} \leq 1$, we can choose $p_{i} \in(0,1)$ satisfying that $R_{h h}\left(\mathcal{C}_{h}^{i}\right)=R_{h l}\left(\mathcal{C}_{l}^{i}, p_{i}\right), \pi_{h l}^{i}=B\left(\theta_{l}^{i}, \beta_{h}, c\right) \pi_{l l}^{i}$ and $\pi_{h h}^{i}=1-\pi_{h l}^{i}$. Otherwise, we choose $p_{i}=0$ and $\pi_{l h}^{i}$ and $\pi_{l l}^{i}$ satisfying that $\pi_{l h}^{i}+\pi_{l l}^{i}=1$ and $\pi_{h h}^{i} R_{h h}\left(\mathcal{C}_{h}^{i}\right)+$ $\pi_{h l}^{i} R_{h l}^{\prime}\left(\mathcal{C}_{l}^{i}\right)=\max \left\{R_{h h}\left(\mathcal{C}_{h}^{i}\right), R_{h l}^{\prime}\left(\mathcal{C}_{l}^{i}\right)\right\}$. Hence, the strategies we have just constructed satisfies (13)-(15) and (17) i.e., it is a continuation equilibrium. Moreover, the allocation $\mathcal{Q}^{2}=\left\{\mathcal{C}_{h}, \mathcal{C}_{l}, p_{i}, \pi_{h h}^{i}, \pi_{h l}^{i}, \pi_{l h}^{i}, \pi_{l l}^{i}\right\}$ satisfies (18), (19), (21) and (22). If (20) is also satisfied, we have that $\mathcal{Q}^{2}$ is feasible in $\psi_{3}\left(\beta_{h}, c, \bar{R}_{h h}, 0\right)$ and $\max \left\{R_{l l}\left(\mathcal{C}_{l}^{i}\right), R_{l h}\left(\mathcal{C}_{h}^{i}\right)\right\} \leq \psi_{3}\left(\beta_{h}, c, \bar{R}_{h h}, 0\right)=R_{l l}\left(\mathcal{C}_{l}^{\prime}\right)$. Therefore, we arrive at a contradiction and $\Omega_{i}^{\prime}<0$.

- When $R_{h h}\left(\mathcal{C}_{h}^{i}\right)<\bar{R}_{h h}$ Similar methods can be used to tackle this case.

Thus, we have finished the proof of the first item of Theorem 5. Its the second item can be proved in similar manner as before. Hence, we get the conclusions in Theorem 5 . 